\title{
Repeated biopsy in the detection of prostate cancer: When and how many cores
}

\author{
Vincenzo Scattoni, Andrea Russo, Ettore Di Trapani, Umberto Capitanio, \\ Giovanni La Croce, Francesco Montorsi \\ Department of Urology, University Vita-Salute, Scientific Institute San Raffaele, Milan, Italy
}

\begin{abstract}
Summary Purpose: We performed an analysis of the literature about the optimal prostate biopsy (PBX) scheme in the repeated setting Methods: We performed a clinical and critical literature review by searching Medline Database from January 2005 up to January 2014. Electronic searches were limited to the English language. The keywords were: prostate cancer, prostate biopsy, transrectal ultrasound, transperineal prostate biopsy.

Results: The recommended approach in repeated setting is still the extended scheme (EPBX) (12 cores). An approach with more than 12 cores according to the clinical characteristics of the patients may optimize cancer detection. Saturation PBX (> 20 cores) clearly improves cancer detection if clinical suspicion persists after previous negative biopsy. Nevertheless international guidelines do not strongly recommended SPBX in all situations of repeated setting. EPBX or SPBX may be, in the future, substituted by multiparametric MRI-targeted biopsies.

Conclusions: Since the scenario in which a PBX is changing, the issue about the number and location of the cores in $P B X$ is still a matter of debate in repeated setting. At present, EPBx are still the gold standard even if SPBX seems to be necessary in many cases. However, random $P B x$ does not represent the approach of the future, but rather imaging targeted biopsy.
\end{abstract}

KeY WORDS: Prostate cancer; Prostate biopsy; Transrectal ultrasound; Ttransperineal prostate biopsy.

Submitted 3 October 2014; Accepted 31 October 2014

\section{INTRODUCTION}

Management of patients with negative biopsy often presents a dilemma. Urologists know well that a negative biopsy does not mean the absence of cancer, and a second biopsy is one of the options. The saturation prostate biopsy (SPBx) was initially introduced to improve prostate cancer (Pca) detection rates (DR) in the repeat setting because initial 10- to 12-core biopsy schemes may miss almost a third of cancers (1).

Nevertheless, the most efficient scheme with the optimal number and location of cores has not been defined yet (1). It is not clear when and how to perform a second biopsy, whether it is necessary to perform the same sampling pro- tocol in each patient, or whether to modify the protocol for different clinical situations. Moreover, it is still controversial whether the detection rate may increase with additional biopsies or whether it is necessary to modify the locations where the cores are taken $(1,3)$.

\section{Methods}

We performed a clinical and critical review of electronic databases by searching Medline, Web of Knowledge and the Cochrane Library from January 2005 up to January 2014 to identify all relevant studies. Electronic searches were limited to the English language, and the keywords prostate cancer, prostate biopsy, transrectal ultrasound, transperineal prostate biopsy were used. Two independent authors performed all aspects of the search strategy, screening the titles and abstracts of all articles and then reviewing the full-text articles in detail.

\section{Prostate biopsy Strategy in the Repeat Setting}

Candidates to repeat $\mathrm{PBx}$ include patients with a prior negative $\mathrm{PBx}$ but with a persistent suspicion of $\mathrm{PCa}$ on the basis of repeated PSA values and/or DRE findings (and other markers such us \%FPSA, complexed PSA, PSAD, PSA velocity and urinary PCA3 score), previous peculiar hystological diagnosis (such as atypical small acinar proliferation of prostate - ASAP or high-grade prostatic intraepithelial neoplasia - HGPIN), candidates to active surveillance or to focal therapy. How and how many cores should be taken in these different scenarios is still unclear and schemes may significantly change in the different patients. Based on the findings that even initial extended PBx (EPBx) miss almost a third of cancers, a SPBx has been adopted to improve PCa DR in patients with suspicious clinical findings following previous negative standard PBx.

There is now good evidence in the literature that SPBx are superior than EPBx in this setting. Zaytoun et al. reported their experience at Cleveland Clinic where they compared EPBx with SPBx in a clearly defined, heterogeneous population of patients undergoing repeat biopsy after a single prior biopsy that failed to diagnose PCa (2). They showed that office-based SPBx significantly increases DR in repeat biopsy compared to EPBx. SPBx detected almost onethird more cancers. For patients with benign initial biop- 
sy, SPBx demonstrated significantly greater PCa detection. For previous ASAP and/or HGPIN, a trend for higher PCa DR was demonstrated in the saturation group but did not reach statistical significance.

Similarly, Scattoni et al. recently tried to identify the optimal combination of sampling sites (number and location) to detect $\mathrm{PCa}$ in patients previously submitted to an initial negative prostatic biopsy (3). They prospectively performed a transrectal ultrasound (TRUS)-guided systematic 24-core PBx in 340 consecutive patients after a first negative biopsy (at least 12 cores). Subsequently, they set the cancer-positive rate of the 24-core PBx at $100 \%$ and calculated PCa DR for 255 possible combinations of sampling sites. They reported that the more cores taken, the higher the cancer DR. They showed a continuum of improvement of the cancer DR when increasing the number of cores, even if the cancer DR of the 24 cores was significantly higher than only the mean DR rates of 14-core schemes. Moreover, at a given number of cores, the DR rates varied significantly according to the different combination of sites considered. All of these studies demonstrate that SPBx provides a higher cancer DR than the extended approach in the repeat setting and that the higher the number of cores, the higher the number of cancers detected.

Nevertheless the regular use of SPBx in clinical practice is not approved (4-5). The National Comprehensive Cancer Network (NCCN) suggests performing a second extended protocol after an initial negative extended scheme and suggests considering SPBx only in patients with a high risk of cancer after multiple negative biopsies. The 2013 European Association of Urology (EAU) guidelines on $\mathrm{PCa}$ do not indicate the template that should be used. Consequently, the ideal strategy for a second PBx procedure has yet to be fully elucidated.

Recently, interest has increased in defining more efficient biopsy schemes for PCa detection with the minimum number of cores. Different variables, both clinical and not clinical, may have an impact on the cancer DR. Apart from the clinical characteristics of the patients, some procedural characteristics may have an even greater impact on the cancer DR. Intuitively, adding more biopsies to prostatic areas not sampled by common extended schemes should increase the DR. It should be noted, however, that increasing the number of biopsy cores is not the solution to the problem and that the relationship between the number of biopsy cores and the resulting cancer DR does not correlate linearly. As a matter of fact, the curve of saturation tends to plateau, and the increase of cores taken in the template is not equivalent to the increase of cancer detected.

Kawakami et al. analyzed the PCa detection rate by using a three-dimensional (3D) 26-core systematic super-EPBx protocol (6). In these analyses, subset biopsy schemes were determined by recursive partitioning to achieve a maximum cancer detection rate at a given number of biopsy cores through a single transrectal approach, a single transperineal approach, or a 3D combination of transrectal and transperineal approaches. They were able to extract a 3D 14-core biopsy protocol that could detect 95\% of cancers with the fewest number of cores. Nevertheless, their approach has the disadvantage of requiring general anesthesia to perform the double approach (transrectal and transperineal). Moreover, they have not specified the most advantageous biopsy protocol according to the clinical characteristics of the patients.

All of these data demonstrate that cancer detection is influenced not only by the number of cores but also by the exact location of the cores.

The report by Delongchamps et al. is a reminder that the urologist needs to do a better job of biopsying the prostate (7). A fairly extensive 36-core biopsy performed in 48 autopsied prostates (median volume: $35 \mathrm{ml}$ ) missed 5 of $12(42 \%)$ cancers found on whole-mount pathologic analysis. In fact, the 36-core biopsy offered no benefit over an 18-core protocol in terms of PCa detection (7). Adopting a scheme that is able to maximize the DR with the fewest number of cores represents a possible new modality of performing PBx. This approach is clinically preferable to adopting a saturation scheme that is unable to increase the cancer DR with the same proportion of increasing numbers of cores. Scattoni et al. recently demonstrated that both the number and the location of biopsy cores taken affect cancer DR in a repeated biopsy setting (3). They also showed that the "optimal" repeat biopsy scheme varies according to the clinical characteristics of the patients. Analysis revealed that for patients with previous ASAP diagnosis, the most advantageous scheme was a combination of a 14-core biopsy (without TZ biopsies). For patients with no previous ASAP diagnosis and percentage of free prostate-specific antigen (\%fPSA) of $10 \%$ or less, the most advantageous scheme was a 14-core biopsy (including four TZ biopsies). The most advantageous sampling scheme for patients with no previous ASAP and \%fPSA greater than $10 \%$ was a combination of a 20-core biopsy (including 4 TZ biopsies). Moreover, the number of repeated biopsy is controversial, also because the DR is inversely related to the subsequent procedure.

Djavan et al. reported in 2001 an original work on the risk of PCa on repeat biopsies performed 6 weeks after an initial negative set. These investigators found that cancer detection rates on biopsies 1, 2, 3 and 4 were $22 \%, 10 \%$, $5 \%$ and $4 \%$, respectively, and that $58 \%, 60.9 \%, 86.3 \%$ and $100 \%$ of patients who had RP had organ confined disease on biopsies 1, 2, 3 and 4. The investigators concluded that biopsy 2 in all cases of a negative finding on biopsy 1 seems justified (8). Similarly, Campos-Fernandes et al. in a cohort with extended biopsies found that $18 \%, 17 \%$, and $14 \%$ of patients had $\mathrm{PCa}$ in second, third, and fourth biopsies, respectively. PCa detected at these sets of biopsies was significant in $85 \%$ of cases (9). Detection of clinically insignificant $\mathrm{PCa}$ (according to Epstein's criteria) is an inevitable risk of repeat biopsy, and its association with the number of biopsy cores is an issue of considerable debate. Moreover, SPBx has been evaluated as a staging tool to improve the characterization of low-volume and well-differentiated PCa, but whether SPBx improves prediction of tumor insignificance remains open to debate. It should be also noted that, in general, cancer missed on initial prostate biopsy is likely to be smaller or more insignificant than those cancers identified on first attempt. In this context, the real issue with PCa detection is not overdiagnosis, since only diagnosis or misdiagnosis exist, but rather potential overtreatment.

Detection and treatment of PCa should always be considered independent processes, and concern about overdetec- 
tion must be weighed against the risk of missing clinically significant cancers.

Finally, in patients with a diagnosis of $\mathrm{PCa}$ candidate to active surveillance SPBx is preferable even if not mandatory, while in cases of focal therapy SPBx may not be sufficient and considered a surrogate to transperineal grid template biopsy. However, the optimal number and location of prostate biopsies in patients in active surveillance with a low grade and low volume PCa and patients who are candidate to focal therapy has not been established.

Several benefits appear to be associated with an image guided approach to prostate biopsy. In summary, fewer men are biopsied overall, a greater proportion of men with clinically significant prostate cancer are biopsied, and fewer men are attributed a diagnosis of clinically insignificant.

Even if the randomized EPBX and SPBx remain the gold standard, many patients demand advances beyond the "old-fashioned" randomized biopsy, which is not considered the "future". Nowadays, multiparametric MRI (mpMRI) has demonstrated to have a high degree of accuracy for the detection of clinically significant prostate cancer and can be used to define a target area before prostate biopsy. In the last five years, the role of image-guided targeted biopsy has grown. The likelihood of detecting cancer in such a visible lesion is definitely higher than with a randomized biopsy if the detection rate per core is considered. MpMRI-targeted biopsies have demonstrated superiority over systematic randomized biopsies for the detection of clinically significant disease and representation of disease burden, while deploying fewer cores. There is evidence that the Gleason score obtained in a targeted biopsy reflects the true Gleason score better than the Gleason score obtained by a randomized PBx (10). Hambrock et al. have demonstrated that MRI-guided biopsies significantly improve pretreatment risk stratification by obtaining cores that are representative of the true Gleason grade (11). In a recent review about MpMRI-targeted biopsies, men with a clinical suspicion of prostate cancer, a biopsy of the prostate that used MRI to inform the sampling was associated with a detection rate of clinically significant prostate cancer of $42 \%$. This approach might permit a reduction in the number of men who need to undergo biopsy if they are deemed to have a normal MRI. The efficiency of the targeted sampling appeared superior to the standard approach (70\% vs $40 \%$ ). Since the randomized PBx was associated with a diagnosis of insignificant prostate cancer in 10\% of men biopsied, this cancer diagnosis might have been avoided if men had undergone targeted biopsy alone (11). The authors also concluded that adopting MpMRI-targeted biopsies rather than randomized PBX, fewer men are biopsied overall, a greater proportion of men with clinically significant prostate cancer are biopsied, and fewer men are attributed a diagnosis of clinically insignificant prostate cancer. On the contrary, other authors have shown that in cases combining targeted and randomized biopsies during one PBx session, a substantial number of cancers were detected in only the randomized cores (12). Relying on the targeted biopsy alone would have led to a significant rate of underdetection in these studies. There is no doubt that EPBx might better characterize PCa volume and cancer extent than just a targeted biopsy: the positive cores give us information on not only the cancer extent but also the number of negative cores. Targeted biopsies seem to reflect the true Gleason score, yet they might underestimate the extent of the cancer. Probably the combination of both targeted and extended biopsies will show the most appropriate information about the correct cancer characteristics.

\section{Conclusions}

The issue about the number and location of the cores is still a matter of debate in repeat setting also because the scenarios in which $\mathrm{PBx}$ is required are changing. At present, SPBx seems to be necessary in most of the cases. However, random prostate $\mathrm{PBx}$ do not represent the future while imaging target biopsy are becoming more popular.

\section{REFERENCES}

1. Scattoni V, Zlotta S, Montironi R, et al. Extended and saturation prostatic biopsy in the diagnosis and characterisation of prostate cancer: a critical analysis of the literature. Eur Urol. 2007; 52:1309-1322.

2. Zaytoun OM, Moussa AS, Gao T, et al. Office based transrectal saturation biopsy improves prostate cancer detection compared to extended biopsy in the repeat biopsy population. J Urol. 2011; 186:850-4.

3. Scattoni V, Raber M, Capitanio U, et al. The optimal rebiopsy prostatic scheme depends on patient clinical characteristics: results of a recursive partitioning analysis based on a 24-core systematic scheme. Eur Urol. 2011; 60:834-41.

4. Scattoni V, Maccagnano C, Capitanio U, et al. Random biopsy: when, how many and where to take the cores? World J Urol. 2014; 32:859-69.

5. Ukimura O, Coleman JA, de la Taille A, et al. Contemporary role of systematic prostate biopsies: indications, techniques, and implications for patient care. Eur Urol. 2013; 63:214-30.

6. Kawakami S, Okuno T, Yonese J, et al. Optimal sampling sites for repeat prostate biopsy: a recursive portioning analysis of threedimensional 26-core systematic biopsy. Eur Urol. 2007; 51:675-83.

7. Delongchamps NB, de la Roza G, Jones R, et al. Saturation biopsies on autopsied prostates for detecting and characterizing prostate cancer. BJU Int. 2009; 103:49-54.

8. Djavan B, Ravery V, Zlotta A, et al. Prospective evaluation of prostate cancer detected on biopsies 1, 2, 3 and 4: when should we stop? J Urol. 2001; 166:1679-83.

9. Campos-Fernandes JL, Bastien L, Nicolaiew N, et al. Prostate cancer detection rate in patients with repeated extended 21-sample needle biopsy. Eur Urol. 2009; 55:600-6.

10. Moore CM, Robertson NL, Arsanious N, et al. Image-guided prostate biopsy using magnetic resonance imaging-derived targets: a systematic review. Eur Urol. 2013; 63:125-40.

11. Hambrock T, Hoeks C, Hulsbergen-van de Kaa C, et al. Prospective assessment of prostate cancer aggressiveness using 3-T diffusion-weighted magnetic resonance imaging-guided biopsies versus a systematic 10-core transrectal ultrasound prostate biopsy cohort. Eur Urol. 2012; 61:177-84.

12. Kuru TH, Roethke MC, Seidenader J, et al. Critical evaluation of magnetic resonance imaging targeted, transrectal ultrasound guided transperineal fusion biopsy for detection of prostate cancer. J Urol. 2013 ; 190:1380-6.

\section{Correspondence}

Vincenzo Scattoni, MD (Corresponding Author) scattoni.vincenzo@hsr.it

Andrea Russo, MD - Ettore Di Trapani, MD - Umberto Capitanio, MD Giovanni La Croce, MD - Francesco Montorsi, MD

Department of Urology, University Vita-Salute, Scientific Institute H San Raffaele. Via Olgettina 60 - 20132 Milan, Italy 